\title{
El problema de la verdad y la alhqeia
}

Roberto Estrada Olguín Universidad Nacional Autónoma de México

\section{Abstract}

The claim of this work is to expose my vision to near problematic of the truth in the scope of the philosophy. For it we will distinguish the two general orientations in whose direction the problem of the truth in the philosophical scope has been boarded: 1) justificationist and 2) ontological. The criterion that allows us to make this distinction is the "attitude and the answer" that have been adopted as opposed to the skepticism. Therefore, in which it follows we will develop the theoretical origins, basic premises and consequences of two attitudes and two answers corresponding to these attitudes as opposed to the skepticism.

In the direction which we denominated justificationist we included the "theories of the truth" that in these times have proliferated, because of them have adopted the same "attitude" and have provided the same "answer" in face of skepticism. For the ontological perspective we will expose the thought of Heidegger on the subject of the truth, because there we find the exposition of the subject adopting a "attitude" and providing a "answer" that is not directed to the search of a justification. These two directions, in my opinion, provide sufficient a minimum sketch of the problem of the truth.

Keywords: truth, objectivity, skepticism, justificationism, alhqeia. 


\section{Resumen}

La pretensión de este trabajo es exponer mi visión acerca de la problemática de la verdad en el ámbito de la filosofía. Para ello distinguiremos las dos orientaciones generales en cuya dirección se ha abordado el problema de la verdad en el ámbito filosófico: 1) la justificacionista y 2) la ontológica. El criterio que nos permite hacer esta distinción es la "actitud y la respuesta" que se han adoptado frente al escepticismo. Por lo tanto, en lo que sigue desarrollaremos los orígenes teoréticos, presupuestos y consecuencias de dos actitudes y dos respuestas correspondientes a estas actitudes frente al escepticismo.

En la orientación que denominamos justificacionista incluimos las "teorías de la verdad", las cuales han proliferado en estos tiempos porque ellas han adoptado la misma "actitud" y han proporcionado la misma "respuesta" ante el escepticismo. Para la perspectiva ontológica expondremos el pensamiento de Heidegger sobre el tema de la verdad, porque en él encontramos el planteamiento del asunto adoptando una "actitud" y proporcionando una "respuesta" que no está dirigida a la búsqueda de una justificación. Además, que esta perspectiva es excluida de las teorías de la verdad, por lo cual su análisis es necesario para determinar su pertinencia en el problema de la verdad. Estas dos orientaciones, a mi juicio, proporcionan un bosquejo mínimo suficiente del problema de la verdad.

Palabras clave: verdad, objetividad, escepticismo, justificacionismo, alhqeia.

\section{Introducción}

T a pretensión de este trabajo es exponer mi visión acerca de la Lproblemática de la verdad en el ámbito de la filosofía. Para ello distinguiremos las dos orientaciones generales en cuya dirección se ha abordado el problema de la verdad en el ámbito filosófico: 1) la justificacionista y 2) la ontológica. El criterio que nos permite hacer esta distinción es la "actitud y la respuesta" que se han adoptado frente al escepticismo. Por lo tanto, en lo que sigue desarrollaremos 
los orígenes teoréticos, presupuestos y consecuencias de dos actitudes y dos respuestas correspondientes a estas actitudes frente al escepticismo.

Por justificacionismo entiendo todo intento de encontrar un criterio o criterios que permitan justificar metodológicamente las creencias de una persona. Dicho de manera diferente, entiendo que el justificacionista es aquel que tiene la "actitud" de tomar en serio al escéptico y que ha decidido, en oposición al escéptico, que el conocimiento existe, que es alcanzable por el hombre y que es comunicable. Pero como tiene al escéptico de oponente, para dar satisfacción a éste no basta con que acepte la existencia del conocimiento, debe demostrar la existencia de dicho conocimiento, que el hombre lo alcanza y que lo comunica. Por ello, su "actitud" ante el escepticismo lo ha conducido al "tipo de respuesta" que derrocaría definitivamente los argumentos del escéptico: encontrar el camino (método) que demuestre la existencia del conocimiento. En la orientación que denominamos justificacionista incluimos las teorías de la verdad analizadas por Apel, porque todas ellas han adoptado la misma actitud y han proporcionado el mismo tipo de respuesta ante el escepticismo. Para la perspectiva ontológica expondremos el pensamiento de Heidegger - el cual es explícitamente excluido por Ape- sobre el tema de la verdad, porque en este pensamiento encontramos el planteamiento del asunto adoptando una actitud y proporcionando una respuesta que no está dirigida a la búsqueda de una justificación. Debemos aclarar que, como el propio Apel menciona, el pensamiento de Heidegger sobre la verdad no es una "teoría de la verdad", pero ello no es suficiente para determinar su pertinencia en el problema de la verdad. Estas dos orientaciones, a mi juicio, proporcionan un bosquejo mínimo suficiente del problema de la verdad.

Para intentar alcanzar nuestro objetivo desarrollaremos la primera orientación iniciando con la explicación de dos presupuestos de las teorías de la verdad: 1) la búsqueda de un fundamento del conoci- 
miento, el cual abordaremos analizando la comprensión del método, como problema fundamental de la epistemología moderna, desarrollo en el cual determinaremos el origen cartesiano de la pretensión de encontrar la justificación última, y 2) el enfrentamiento con el escepticismo en el cual se encontró el empirismo, la teoría del conocimiento y la filosofía de la ciencia de finales del siglo XIX y principios del XX, debido a un retorno a Hume. Después, de manera muy breve, fijaremos lo que se comprende por escepticismo para pasar enseguida a determinar el lugar donde esta primera orientación ubica el fenómeno de la verdad: las proposiciones. Luego, expondremos el problema del regreso infinito como la forma de tratar el problema de la verdad por parte de las llamadas teorías de la verdad. A continuación, comentaremos brevemente qué se entiende por objetividad dentro de esta perspectiva, señalando que objetividad sólo se puede entender frente a subjetividad. Y decimos frente y no contra con la intención de que no se perciban como conceptos opuestos, pues como veremos pueden resultar hasta sinónimos. Mostraremos que objetividad es entendida como intersubjetividad. Para finalizar, expondremos en esta primera orientación la explicación sobre la actitud adoptada y la respuesta proporcionada por ella ante el escepticismo.

Para desarrollar la postura de la segunda orientación, la orientación ontológica, iniciaremos con el análisis de la postura del escepticismo mediante la discusión sobre la primacía de la pregunta ¿existe la verdad? Posteriormente, explicaremos por qué el punto de partida del problema de la verdad necesariamente es la pregunta ¿qué es la verdad? y aclararemos el sentido de ambas preguntas. Luego, intentaremos explicar dónde ubica Heidegger el fenómeno de la verdad: en el ámbito ontológico del ente que pregunta ¿qué es la verdad? Enseguida, expondremos cómo es comprendida la verdad como condición ontológica del ente que pregunta sobre ella: la $\alpha \lambda \eta \theta \varepsilon \iota \alpha$. Para finalizar, explicaremos los diferentes tipos de verdad que encontramos en el pensamiento de Heidegger y explicaremos cómo se relacionan 
entre sí esos tipos de verdad y cómo se entiende la objetividad en este marco de pensamiento. Y, por último, terminaremos intentando mostrar cómo se relacionan ambas orientaciones.

\section{El "escándalo" de la filosofía y las teorías de la verdad}

1. Dos presupuestos de las teorías de la verdad

\section{A. El presupuesto de la búsqueda de un fundamento}

En el Discurso del método vemos claramente cómo se trata de derrocar a la autoridad ${ }^{1}$ con una noción que Descartes heredó de la antigüedad. ${ }^{2}$ El Discurso inicia señalando que "le bon sens ou la raison est naturellement égale dans tous les hommes”, lo cual tiene la intención

\footnotetext{
1 "Está fuera de duda la importancia de Montaigne en el traspaso del espíritu escéptico de los antiguos al pensamiento moderno de Descartes y en el derrocamiento de la autoridad y de la metafísica eclesiástica” (Dilthey, 1978: 47). Este señalamiento únicamente pretende poner de relieve que Descartes tiene una herencia que no puede negar. Esa herencia fue, precisamente, legada por el Renacimiento y la tradición hermética a través de la Compañía de Jesús y su enseñanza en La Flèche. La descripción y explicación de la enseñanza impartida en la época en que Descartes estuvo en La Flèche puede verse Salvio Turró (1985: 183-220).

2 "Con el Renacimiento renacen los epicúreos, los estoicos, los panteístas embriagados de naturaleza, los escépticos y los ateos. Y, por primera vez, aparecen entre los jóvenes pueblos romano-germánicos, a la luz del día y con la visera levantada, todos los matices del temple vital y de la fe" (Dilthey, 1978: 27). Además, puede verse la detallada y amplia descripción de la diferencias entre la concepción de la Edad Media, la del Renacimiento y de la Modernidad, descritas por Salvio Turró (1985). En esta obra, el autor distingue la concepción aristotélica de la Edad Media (como una explicación biológico-teleológica del mundo, dado el papel que juegan los fines) de la concepción del Renacimiento, la cual es una explicación animista (el macro y microcosmos como organismos) y de la concepción matemática de la naturaleza de la modernidad.
} 
de poner énfasis en que el error y la falsedad no se producen por ser unos hombres más razonables que otros, es decir, porque algunos individuos poseen la revelación de la verdad y otros no; sino por el hecho de que conducen la razón por caminos (métodos) diferentes. De ese modo, pues, la tarea que pretende Descartes es:

[...] y así, me liberaré poco a poco de muchos errores que pueden ofuscar nuestra lumiére naturelle y hacernos menos capaces de escuchar a la razón. Pero, después que he empleado algunos años a estudiar en el libro del mundo y tratar de adquirir alguna experiencia, he tomado, un día, la resolución de estudiar también en mí mismo y de emplear todas las fuerzas de mi espíritu para elegir los caminos que debía seguir (1960: 23).

Esa lumiere naturelle o bon sens o raison, es la naturae lumen o ratio naturalis de los estoicos. Aquí no se trata de responder al escéptico, sino de derrocar una autoridad y de establecer que la verdad del conocimiento no está en el "libro del mundo", ${ }^{3}$ sino en uno mismo. Además, Descartes ha puesto de manifiesto que el verdadero problema del conocimiento es el problema del método: "Car ce n'est pas assez d'avoir l'esprit bon, mais le principal est de l'appliquer bien" (1960: 18).

En su carta dirigida a Mersenne, fechada en marzo de 1637, Descartes señala que no escribió un Tratado del método sino un Discurso del método con la finalidad de hacer notar que su objetivo no es "enseñar" un método sino sólo hablar sobre él. ${ }^{4}$ No tenemos razones

${ }^{3}$ Ese "derrocamiento de la autoridad y de la metafísica eclesiástica", vino precisamente a instaurar el pensamiento moderno. Esa autoridad se había establecido como portadora de la verdad, en general, y de la verdadera interpretación de las Sagradas Escrituras, en particular. En efecto, puesto que la verdad era revelación ésta era exclusiva de ciertos individuos privilegiados, iluminados.

${ }^{4}$ Citado por Jacob Klein, (1968: 283, nota número 278). Esto se complementa perfectamente con lo que Descartes dice al principio de la primera parte del Discurso: "Ainsi mon dessein n'est pas d'enseigner ici la méthode que chacun doit 
suficientes para poner en duda la sinceridad de Descartes cuando dice que su objetivo no es enseñar un método. En efecto, lo primero no es enseñar un método, sino antes es menester mostrar la propia necesidad de un método para la obtención del conocimiento $\mathrm{y}$, por tanto, la tarea siguiente es encontrar ese método.

Por tanto, en Descartes la "necesidad" de un método seguro y certero es el primer conocimiento que se adquiere y no el cogito ergo sum; método que debe encontrar un punto firme y seguro, el cual se busca en uno mismo después de intentar encontrarlo en el mundo y fracasar en ese intento y convencerse de que por esa vía se fracasará. Ahora bien, ese método se identifica con la mathesis universalis. Ésta es el propio orden y medida que constituye la naturaleza de la razón humana; de este modo, la racionalidad se relaciona estrechamente con la idea del método y con la perspectiva de la justificación $n^{5}$ del conocimiento.

Descartes habla de una ciencia distinta a las matemáticas ordinarias, ciencia que, además, deberá contener los rudimentos de la razón humana, es decir, la racionalidad misma, e identifica el método con ciencia y ésta con la mathesis universalis. ${ }^{6}$ Claramente, pues, Descar-

suivre pour bien conduire sa raison, mais seulement de faire voir en quelle sorte j'ai tâché de conduire la mienne"(1960: 19).

5 "Aunque en este tratado hable con frecuencia de figuras y números [...] el que siga con atención mi pensamiento observará fácilmente que no es mi objetivo hablar de las matemáticas ordinarias sino exponer otra ciencia [...]”.

"Esta ciencia debe contener los primeros rudimentos de la razón humana y servir además para extraer de un objeto cualquiera las verdades que encierre. Hablando con sinceridad he de confesar que ella es preferible a todos los conocimientos que los hombres nos han transmitido, porque es la fuente de donde brotan aquellos" (Descartes, 1971: 102).

6 "Método y no otra cosa parece lo que se designa con el extraño nombre de álgebra, con tal que se prescinda de la multiplicidad de números y figuras inexplicables que lo oscurecen". Y más adelante: "Reflexionando sobre esto más atentamente 
tes identifica el método con lo que en su época se denominaba álgebra, y ésta, a su vez, es la ciencia general del orden y la medida, la cual él llama con su nombre usual y antiguo de matemáticas universales. Además, notemos que "ciencia general" significa el tratamiento del orden y la medida con abstracción de toda materia particular; por todo ello se habla de Reglas para la dirección del espíritu, pues el método consiste en dirigir el espíritu por el camino correcto.

Ahora bien, lo relevante de todo esto es que el espíritu necesita dirección, necesita conducirse por la senda correcta, necesita método - sea algorítmico o no-y lo necesita porque la distinción entre lo verdadero y lo falso no depende de que unos hombres sean más razonables que otros, sino de conducir por caminos diferentes el bon sens, que es por naturaleza igual en todos los hombres. Esta "democracia" de la razón es el presupuesto de la necesidad de un método.

Por otra parte, la duda cartesiana está motivada por la necesidad de asegurarse con certeza de la verdad del conocimiento, puesto que para Descartes el conocimiento consiste en estar en condiciones de demostrar que se conoce; esto es, decir que alguien conoce significa no sólo que éste llegue a tener creencias verdaderas, sino que se necesita también poder proporcionar el fundamento de dichas creencias. ${ }^{7}$ De tal manera que el primer paso de ese método es encontrar un punto arquimedeano que nos permita levantar el mundo.

descubro que debemos referir a las matemáticas todas las cosas en que se examina el orden o la medida, importando poco se trate de números, figuras, astros, sonidos, o de cualquier otro objeto si se investiga esa medida u orden. Debe, pues, existir una ciencia general que explique todo lo que podamos conocer relativo al orden y a la medida sin aplicación de ninguna materia en especial. La denominación de esta ciencia no consiste en un nombre extranjero, sino en el antiguo y usual de matemáticas universales" (Descartes, 1971: 103).

7 "Los mortales tienen en ocasiones una curiosidad tan ciega que dirigen su espíritu por vías desconocidas, sin ninguna esperanza y únicamente por ver si la casualidad les depara lo que buscan, a semejanza del que devorado por el insensato deseo de 
Es claro, pues, que a pesar de la posibilidad de llegar a adquirir alguna verdad, por azar o por casualidad, eso no basta para poder decir que se conoce, porque quien así ha llegado a tener alguna verdad no puede proporcionar el fundamento de ella, ni podrá estar seguro y cierto de que está ante una verdad. Charles B. Guignon ha expresado de manera muy clara esta idea de la siguiente manera:

El cartesiano no está preocupado solamente con cómo ocurre efectivamente que llegamos a las creencias que tenemos. Esta cuestión fáctica - a quaestion facti- es irrelevante, desde que puede ser el caso que creemos ciertas cosas en virtud de nuestro código genético o a través de alguna otra cadena causal totalmente externa a la verdad de la creencia. Lo que es del interés del sentido común y del cartesiano, no es la historia de cómo llegamos a adoptar nuestras creencias, sino qué nos justifica en adoptar esas creencias -la quaestio juris (Guignon, 1983: 33). ${ }^{8}$

El método para Descartes no sólo es la ciencia general del orden y la medida, sino también es la única forma de acceder a la quaestio juris, la cual es la cuestión que interesa al cartesiano, puesto que la

descubrir un tesoro, recorriese sin cesar todos los caminos por si algún viajero lo hubiese enterrado en uno de ellos. Así estudian casi todos los químicos, y la mayor parte de los geómetras y muchos filósofos.

"No niego que en medio de sus errores tengan a veces la fortuna de encontrar alguna verdad; pero no por eso son más hábiles; en todo caso serán más afortunados. Mejor que buscar la verdad sin método es no pensar nunca en ella, porque los estudios desordenados y las meditaciones oscuras turban las luces naturales de la razón y ciegan la inteligencia” (Descartes, 1971: 100-101).

8 "The Cartesian is not concerned merely with how we actually happen to arrive at the beliefs we hold. This factual question - the quaestio facti- is irrelevant, since it might be the case that we believe certain things by virtue of our genetic coding or through some other causal chain totally extraneous to the truth of the belief. What is of interest to common sense and the Cartesian alike is not the story of how we have come to hold our beliefs, but what justifies us in holding those beliefs - the quaestio juris". 
quaestio facti no es suficiente para la obtención de la verdad. Así, el método o la mathesis universalis se muestra como condición, como el presupuesto de la comprensión del conocimiento, como la justificación de nuestras creencias. ${ }^{?}$

Un número muy considerable de las reflexiones sobre la verdad y la objetividad tiene como uno de sus puntos de partida esta búsqueda del fundamento de nuestro conocimiento — por lo cual podemos llamarla fundamentalista - como pretendemos explicar más adelante. Sin embargo, la preocupación por encontrar una justificación o fundamento de nuestro conocimiento sólo es motivado porque se piensa que aún no se tiene dicho fundamento y que, además, es una necesidad y un deber obtenerlo. En efecto, un segundo punto de partida que ha propiciado las reflexiones modernas sobre la verdad y la objetividad es el escepticismo.

\section{B. El presupuesto de satisfacer al escepticismo}

El escepticismo arribó en el siglo XIX a la filosofía de las ciencias empíricas por un "retorno" a Hume, retorno que se produjo después de una crítica de la filosofía de Kant llevada a cabo por renombrados

\footnotetext{
${ }^{9}$ El "libro del mundo" de la escolástica deja de ser el portador de la verdad, la cual era revelada a los espíritus elevados, para poder "leer" las verdades ocultas en ese libro. El mundo, en Descartes, es puesto en duda preliminarmente y se busca el fundamento no en el "libro del mundo", sino en él mismo, en la conciencia, en el yo, en el cogito. El sujeto viene a establecerse como el fundamento, como lo único seguro clara y distintamente. Descartes tomó la resolución de estudiar en sí mismo, para encontrar el fundamento último de nuestro conocimiento. Pone así al yo como la base, el fundamento último de todo conocimiento. Así es como el conocimiento pasa a ser comprendido como fenómeno de la conciencia. Esto es, sólo es conocimiento aquello que está en la conciencia.
} 
filósofos y científicos al final de dicho siglo. Ernst Mach se preocupó por criticar la distinción kantiana entre cosa en sí y fenómeno. ${ }^{10}$ La influencia de Mach sobre los principales miembros del Círculo de Viena no siempre se ha puesto de relieve de manera suficiente. Henri Poincaré se ocupó de llevar a cabo la crítica de la distinción

${ }^{10}$ Ernst Mach (1838-1916), con su crítica a la ciencia newtoniana, es considerado un precursor de Einstein, contribuyendo así a la ciencia; en filosofía se interpreta como fenomenalista; y finalmente en historia de la ciencia su libro Die Mechanik (1883) se considera imprescindible para el mejor entendimiento de la ciencia moderna. Ernst Mach nos dice en Análisis de las sensaciones (1885): "Hablando propiamente, el mundo no está compuesto de 'cosas' como sus elementos, sino de colores, tonos, presiones, espacios, tiempos, brevemente, lo que comúnmente llamamos sensaciones individuales". Sin embargo, reconocer que el mundo está compuesto de estas sensaciones individuales, es el resultado de un análisis realizado sobre lo que se nos presenta inmediatamente. Pues, según Mach, los colores, sonidos, presiones, etc., se presentan combinadas de diversas maneras; a cada una de estas combinaciones él las llama complejo o tejido compuesto de sensaciones. Es clara la influencia de Hume en esta idea: "Por consiguiente no tenemos ninguna idea de sustancia que sea distinta de la de una colección de cualidades particulares, ni poseemos de ella otro significado cuando hablamos o razonamos sobre este asunto"(1988: 61).

Ahora bien, ciertas sensaciones de estos complejos, combinadas con funciones espaciales y temporales, aparecen como más estables, y a estas sensaciones más estables las designamos con nombres mediante el lenguaje; entonces, aparecen como "cuerpos". La pretensión de analizar, es decir, aislar los elementos constitutivos de los complejos de sensaciones, puede entrar en contradicción con el hábito de designar con nombres lo que se piensa que es estable.

"La imagen vaga de lo estable [dice Mach] que no varía perceptiblemente porque aparezca o desaparezca esta o la otra de las partes que le constituyen, parece ser algo en sí. Como cada una de estas partes puede ser suprimida sin que la imagen deje de representar el conjunto, pudiendo ser siempre éste reconocido, se cree que se podría suprimir todo y que aún quedaría algo. Así, nace naturalmente el concepto de una cosa en sí (reconocida como distinta de su 'apariencia'), que al principio se impone, pero que luego se nos revela como absurdo". 
entre juicios analíticos y juicios sintéticos. ${ }^{11}$ Este aspecto de la filosofía kantiana, que se atribuye también al empirismo lógico, ha sido igualmente criticado por Quine (1962: 49-81) en su célebre artículo

El absurdo o la contradicción es resultado de no percatarse de que los complejos de sensaciones no son estables. Por eso Mach califica de "vaga" la imagen de lo estable. Este no percatarse tiene como consecuencia el que no se reconozca que los complejos de sensaciones son vistos como "cuerpos" sólo por hábito, sólo por adaptación de pensamiento a esos complejos. Dicho en otras palabras, no se reconoce que lo estable no existe en los complejos de sensaciones y que únicamente ha sido introducido por el pensamiento, con el fin de representarse los complejos de sensaciones; sino que al pensar que lo estable existe y que sus elementos constitutivos pueden suprimirse (aisladamente), sin dejar de pensar el conjunto, entonces, surge la apariencia de que la "cosa" o el "cuerpo" son algo distinto de sus partes constitutivas. Por lo cual Mach señala que la cosa, el cuerpo, la materia no es nada fuera de las sensaciones; con lo cual quiere decir que el problema filosófico de una cosa en sí, distinta de su "apariencia" es, en realidad, un pseudoproblema. Es decir, la aceptación de la cosa en sí es "metafísica”. Ahora sí resulta claro el porqué Mach rechaza la metafísica y afirma que el mundo está constituido por sensaciones individuales, y no por cosas.

${ }^{11}$ Henri Poincaré (1854-1912) dirigirá su atención a la naturaleza de los juicios sintéticos a priori que, según Kant, son el fundamento del conocimiento científico, y en especial de los axiomas de la geometría. Poincaré afirmó que los juicios de la ciencia no son ni sintéticos a priori ni juicios empíricos, sino definiciones convencionales, que son propuestas para usarlas como instrumentos científicos. Sin embargo, la palabra convención debe ser tomada con cuidado en el contexto del pensamiento de Poincaré, pues los principios de las ciencias siempre están, para este filósofo, basados en la experiencia, apoyados en la inducción pero no lógicamente deducidos de ella, sino producidos por el espíritu a través de su potencia de generalización de la capacidad heurística del científico: "Los principios de la mecánica se presentan, entonces, para nosotros bajo dos aspectos diferentes. De una parte, son verdades fundadas sobre la experiencia y verificadas de una manera muy cercana al de los sistemas aislados. Por otra parte, son los postulados aplicables al conjunto del universo y vistos como rigurosamente verdaderos.

"Si esos postulados presentan una generalidad y una certeza que hace falta en las verdades experimentales de las cuales han sido inferidas, es porque ellos se reducen, en un último análisis, a una simple convención, que tenemos el derecho de realizar, porque estamos ciertos de que ninguna experiencia vendrá a contradecirla.

"Esta convención no es, por tanto, absolutamente arbitraria; ella no surge de nuestro capricho; nosotros la adoptamos porque ciertas experiencias nos han mostrado que ello sería cómodo" (1970: 162-163). 


\section{"Dos dogmas del empirismo". Por último, Helmholtz y Schlick cri- ticaron la noción de "intuición" kantiana. ${ }^{12}$ \\ Esas críticas de las nociones kantianas y su consecuente retorno a Hume no evitaron sino, por el contrario, provocaron que el empi-}

Poincaré pone primeramente en cuestión la naturaleza del razonamiento matemático aceptada por Kant. Para elucidar esta naturaleza. Poincaré inicia afirmando que existe una contradicción en la posibilidad misma de ese razonamiento: si éste no es deductivo, ¿de dónde le viene el rigor de sus inferencias que nadie puede negar? Por el contrario, si todas las proposiciones de la matemática pueden ser derivadas unas de otras por las reglas de la lógica, ¿por qué no se reduce toda la matemática a una enorme tautología? Por ejemplo, en La ciencia y la hipótesis (1906) señala: "Sin duda nos podemos remontar a los axiomas que son la fuente de todos los razonamientos. Si se juzga que no pueden reducirse al principio de contradicción, si ya no se quiere ver a los hechos experimentales que no pueden participar de la necesidad matemática, se tiene aún el recurso de clasificarlos entre los juicios sintéticos a priori. Esto no es resolver la dificultad, es solamente bautizarla" (10). 12 Según Alberto Coffa, la contribución más importante de Helmholtz (18211894) con relación a la epistemología fue el haber demostrado que el espacio de la geometría no posee ninguna necesidad (ni de la intuitición, ni del entendimiento), sino que la geometría tiene una base empírica en alguna medida. En efecto, Kant distinguió entre una necesidad de la intuición (Anschauungsnothwendigkeit) y una necesidad del entendimiento (Denknothwendigkeit), y el espacio es, según Kant, una intuición y una condición necesaria —es decir, a priori- de posibilidad de la experiencia. De suerte que para Kant, la geometría como ciencia del espacio no puede tener un fundamento empírico. Cfr. "Geometry, pure intuition and the a priori" (1991).

Ahora bien, Helmholtz cambia la noción de "representación", cambio que le sirve para mostrar que si entendemos representación como él la ha definido, entonces la intuición — la cual es una representación — no impone ningún tipo de necesidad a la geometría. Sin embargo, con ese cambio de la noción de representación de Helmholtz está alterando totalmente la noción de intuición dada por Kant: "De la muy mal usada expresión 'representar' o 'ser capaz de pensar cómo algo ocurre', entiendo que podemos describir [depict] la serie de sensaciones-impresiones que podemos tener si tal cosa ocurre en un caso particular. No veo cómo podamos entender algo distinto, sin abandonar el sentido completo de la expresión". 
rismo del siglo XX se viera rodeado de un ambiente de escepticismo. Escepticismo que con la ayuda del convencionalismo de Poincaré, Schlick y Duhem se tradujo en relativismo. Este es el ambiente en el cual este empirismo reflexionó sobre la noción de "verdad". Pero la herencia cartesiana según la cual es necesario encontrar una justificación del conocimiento implícitamente determinó la "actitud" y "respuesta" que se tomó ante ese escepticismo.

Kant definió la intuición (Anschauung), específicamente el espacio y el tiempo, en cuanto intuiciones puras, como formas subjetivas que moldean a priori los datos de los sentidos, formas que no son conceptos del entendimiento, sino que son condición de posibilidad de las "representaciones" conceptuales del entendimiento. Esta definición de intuición no juega ningún rol en la definición de "representación" dada por Helmholtz. De esa manera éste ha cambiado no sólo la idea de representación sino también la de intuición, lo cual se muestra en la noción que proporciona de lo "concebible"; así cuando él habla de "intuicionalmente concebible", debemos entender por concebible "representable", es decir, poder dar la serie de impresiones sensibles que podemos tener en el acto de intuir algo. Sólo así podemos entender que la intuición no impone ninguna necesidad a la geometría, ni a cualquier ciencia. "Si, en efecto, [dice Helmholtz] espacios de otro tipo [distintos al euclideano] son imaginables [representables, concebibles] en el sentido establecido, entonces eso refutará la afirmación de que los axiomas de la geometría son, en el sentido de Kant, consecuencias necesarias de una forma trascendental dada a priori en nuestras intuiciones".

Estas ideas están presentes muy esencialmente en el pensamiento de Schlick (1882-1936) quien, en su famoso libro General Theory of Knowledge (1918), muestra una constante discusión con las ideas kantianas de intuición, cosas en sí mismas, categorías, etc. Citemos un párrafo de dicho libro en el cual se resumen las ideas de Helmholtz: "Nuestro descubrimiento es [dice Schlick], entonces, que la geometría —no sólo como una ciencia conceptual pura sino también como la ciencia del espacio- no procede de proposiciones sintéticas a priori. Por el contrario, procede de convenciones, esto es, de definiciones implícitas. En la medida en que se mueve dentro de esas definiciones y de los teoremas que pueden deducirse rigurosamente de ellas, es puramente analítica en carácter y, por tanto, posee validez absoluta. Pero las proposiciones sobre las relaciones espaciales de la realidad no pertenecen a esta geometría pura; más bien ellas forman parte de su aplicación al material empírico. 
Así pues, el empirismo lógico moderno del siglo XX parte de estos dos supuestos: 1) la búsqueda de un fundamento del conocimiento mediante la determinación de un método, heredado de Descartes, y 2) la necesidad de dar satisfacción al escéptico, presupuestos que se necesitan y se implican recíprocamente. La manera de abordar "el fenómeno de la verdad" depende de la actitud y la respuesta que se tome respecto al escepticismo y, por tanto, de la actitud y la respuesta ante todo relativismo. Pero antes de explicar esta afirmación y con la pretensión de dirigirnos hacia esa finalidad, debemos precisar lo que se entiende por escepticismo.

Hay muchas maneras de exponer lo que se comprende por escepticismo, pero puesto que nuestro tema es la verdad —y la objetividad- lo precisaremos bajo este tema, de tal manera que el problema suele exponerse en su integridad abarcando tres formulaciones de distinta rigidez: 1) que la verdad no existe, 2) que si existe no es captable por el ser humano y 3) que si existe y es captable por el ser humano, es imposible comunicarla a los demás. ${ }^{13}$

La actitud y la respuesta ante el escepticismo así formulado, y de las cuales surgen todas las teorías de la verdad, suelen pretender refutarlo por vía de encontrar un método que deje establecido, de una

Son juicios sobre el comportamiento de las varas de medición y sobre el lugar (locations) de cuerpos. Como tales ellos son sintéticos en carácter pero a posteriori; sólo la experiencia puede determinar su validez".

Así, pues, Schlick acepta que el carácter universal y verdadero de la ciencia (particularmente de la geometría) tiene su origen no en las intuiciones a priori sino en definiciones convencionales. Y los juicios sobre las relaciones espaciales reales siempre son empíricas y, por tanto, a posteriori. De hecho, desde esta nueva perspectiva, no existen $-\mathrm{y}$ no es posible que existan — los juicios sintéticos a priori. Los juicios a priori son analíticos y los juicios sintéticos son empíricos. Para mayores detalles sobre este asunto puede consultarse el propio texto de Schlick ya citado.

${ }^{13}$ Platón ya planteó el problema de manera similar en el libro X de las Leyes, pero con respecto a los dioses aquí se expone la crítica a las teorías siguientes: a) los 
vez por todas, aquello que justifique el conocimiento. Esta actitud y respuesta al escepticismo parten de plantear el problema de una manera determinada, ${ }^{14}$ la cual ya ha decidido implícitamente lo fundamental y la direccionalidad ante el fenómeno de la verdad. Veamos ahora, cómo es que las teorías de la verdad plantean el problema y la solución de la verdad.

\section{Las teorías de la verdad y la objetividad}

El asunto podemos plantearlo mediante el llamado problema del regreso infinito. El punto de partida de este problema es la ubicación de la verdad, es decir, que la verdad se halla en las proposiciones, en los juicios o sentencias. Es decir, que aquello a lo que llamamos verdadero son las proposiciones. Por tanto, para aceptar una proposición como verdadera debemos tener un procedimiento que la justifique, que constituya la razón de que la aceptemos como verdadera. Ahora bien, este procedimiento es un argumento inferencial del cual se deduce la proposición en cuestión. Entonces este procedimiento debe partir de otras proposiciones como premisas.

Evidentemente, para aceptar esas otras proposiciones como verdaderas es necesario que hayan pasado por el proceso justificatorio, el cual deberá tomar como punto de partida otras proposiciones como verdaderas por el mismo procedimiento y así sucesivamente hasta

dioses no existen, b) si existen no tienen poder sobre los asuntos humanos y c) si existen y algo influyen en los asuntos humanos fácilmente se los complace con oraciones. Para demostrar que a) es falsa se plantea que la cuestión depende de qué aceptar como principio, es decir, de si el cuerpo es anterior al alma o viceversa. Y se parte de analizar qué es el movimiento e indagar sus condiciones de posibilidad.

${ }^{14}$ Los elementos que dan esta determinación son los ya expuestos como herencia cartesiana de encontrar aquello que fundamente el conocimiento ante el peligro del escepticismo producto del empirismo de Hume. 
el infinito. De tal modo que no es posible, por este procedimiento, saber si nuestras proposiciones son verdaderas.

Todas las teorías de la verdad están orientadas, directa o indirectamente, a proporcionar una solución a este problema del regreso. No se sabe con precisión cuántas teorías de la verdad hay o puede haber. Pienso que la lista que nos ha proporcionado Karl-Otto Apel en su obra titulada Teoría de la verdad y ética del discurso (1991: 45) ${ }^{15}$ basta para dar una idea de la variedad de ellas y cuya orientación está determinada por el problema que venimos describiendo. Dicha lista es la siguiente:

1. Teoría de la correspondencia

2. Teoría de la evidencia

3. Teoría de la coherencia

4. Teoría pragmatista

5. Teoría semántica de la correspondencia de Tarski

6. Teorías de la correspondencia realista postarskianas

7. Teorías constructivistas del consenso

8. Teoría pragmático-trascendentales del consenso

Como podemos ver, a pesar de que Apel distingue 1, 2, 5 y 6 como distintas, estas cuatro de la lista podemos considerarlas como versiones de una sola: la teoría de la correspondencia; y la 7 y la 8 son dos versiones de una segunda: la teoría del consenso. En efecto,

${ }^{15}$ Excluimos deliberadamente las teorías pragmáticas de la verdad que sí incluye Apel porque se alejan de nuestro objetivo ya que al ser el término justificación un término genérico y el término práctica tiene un significado muy amplio, por lo cual, puede significar justificación moral, social, políticamente u otro sentido extracientífico, es decir, extracognoscitivo. La teoría pragmática de la verdad corre el peligro de entenderse como un procedimiento que justifica el conocimiento desde un punto de vista extracognoscitivo. 
la teoría clásica de la correspondencia tiene por tarea establecer la coincidencia entre los juicios y las cosas en sí, entendidas como independientes del sujeto y completas por sí mismas sin intervención del sujeto; pero como a partir de Kant se acepta que las cosas en sí no son accesibles al conocimiento del hombre (por lo cual la tarea de realizar la correspondencia o coincidencia entre los términos de la relación es imposible) y que sólo puede conocer los fenómenos, entonces la teoría de la evidencia fenoménica se ha propuesto como tarea llevar a cabo la coincidencia o correspondencia entre la evidencia fenoménica y los juicios. Luego, la evidencia fenoménica es relativa debido a que va unida inseparablemente a una interpretación lingüística, por lo cual la teoría semántica de la verdad propone limitar las interpretaciones lingüísticas mediante el establecimiento de un lenguaje artificial formalizado, lenguaje que carecería de las diversidades de los lenguajes cotidianos y que fijaría el significado único, significado independiente de los lenguajes cotidianos y de los sujetos concretos que elaboran los juicios. Con esto se determinaría un mundo semántico independiente de las interpretaciones lingüísticas; así la tarea consistiría en hacer corresponder o en establecer la coincidencia entre los juicios y el mundo semántico construido con ese lenguaje artificial formalizado. Entonces, la teoría clásica de la verdad como correspondencia, la teoría de la evidencia fenoménica y la teoría semántica de Tarski proponen que la verdad consiste en la correspondencia o coincidencia de dos términos, pero proponen diferentes términos para corresponder.

Por otra parte, de tal modo que podamos limitarlas a tres diferentes tipos de teorías: ${ }^{16}$

\footnotetext{
${ }^{16}$ Aunque esta clasificación sigue siendo superficial, pues estos tres tipos de teorías de la verdad suponen una relación de correspondencia o adecuación. En efecto, la coherencia supone la relación de correspondencia entre las cosas que deben ser coherentes. Y el consenso supone la coherencia y la correspondencia entre aquello sobre lo que hay que llegar a un consenso.
} 


\section{La teoría de la correspondencia ${ }^{17}$}

2. La teoría de la coherencia ${ }^{18}$

3. La teoría del consenso ${ }^{19}$

No pretendemos exponer en este escrito el desarrollo de los argumentos y contraargumentos de estas teorías de la verdad, pues eso nos conduciría a involucrarnos en la orientación ya determinada por la manera de plantear el problema desde la perspectiva en la cual están inmersas esas teorías, lo cual nos alejaría del propósito de este trabajo. No obstante, una breve exposición de las soluciones que proponen estas teorías bastará para nuestra finalidad. Sin embargo, estas teorías de la verdad no son mutuamente excluyentes, sino que pueden ser complementarias pero tampoco son idénticas.

La teoría de la correspondencia intenta solucionar el problema del regreso infinito mediante la aceptación de unas proposiciones que funcionen como base (las cuales son producto del consenso, por lo cual un defensor de la teoría del consenso podría argumentar que finalmente la verdad depende de las proposiciones que se aceptan por consenso), pero al no tener un criterio satisfactorio que proporcione razones para decidir cuáles proposiciones deben tomarse como bási-

\footnotetext{
17 Según Apel, en esta teoría están incluidos autores como Aristóteles, Descartes, Brentano y Husserl. Pero bien podemos mencionar también a Kant, Hume, Locke y muchos otros más. Sin embargo, para darse una idea de la orientación de la discusión moderna sobre la verdad, pueden consultarse los siguientes textos: (Tarski, 1944; Gettier, 1963: 121-123; Austin, 1964 y Villoro, 1982).

${ }^{18}$ Apel incluye en esta teoría a autores como Hegel, Nuerath y Rescher, entre otros. Pueden consultarse los siguientes textos: Otto Neurath, "Proposiciones protocolares" y "Sociología en fisicalismo", en Ayer (1959: 205-214 y 287-324) y Rescher, (1991).

${ }^{19}$ Para darse una idea de esta teoría véase el propio texto de Apel ya citado.
} 
cas, propone que el límite de procedimiento inferencial de justificación descansa en la aprehensión inmediata. ${ }^{20}$

La teoría de la coherencia, por su parte, propone una solución a partir de dos tesis. La primera afirma que toda justificación de proposiciones individuales es inferencial y rechaza la existencia de proposiciones básicas. Por tanto, mediante esta tesis no hay fundamentación del conocimiento. La segunda afirma que el regreso infinito debe terminar en un sistema cerrado, volviendo en círculo y su justificación es holística (no individual) y por la coherencia interna del sistema. ${ }^{21}$

La teoría del consenso parte del supuesto de las creencias compartidas, tácita o expresamente, por un grupo o una comunidad. Consiste en la aceptación de un conjunto de proposiciones como verdaderas por parte de ese grupo o comunidad, donde ese conjunto puede o no ser modificado por diversos factores. El consenso también puede ser construido por las reglas lingüísticas aceptadas por la comunidad.

Por otra parte, ¿cómo es determinada la objetividad por estas teorías de la verdad? Antes de intentar responder a esta cuestión, aclaremos qué se comprende por objetividad en general. Dicho en pocas palabras, la objetividad es la cualidad de ser objeto, la esencia de los objetos, aquello que hace que los objetos sean objetos. ${ }^{22}$ Para

\footnotetext{
20 También puede consultarse para una exposición detallada del desarrollo de los argumentos y las objeciones a estas teorías, pero desde una postura anglosajona, el siguiente trabajo: Goldman, 1967: 357-372.

${ }^{21}$ Consúltese los textos de Laurence Bonjour: "The coherence theory of empirical knowledge" (1976: 281-312) y "Can empirical knowledge have a fundation?" (1978: 1-13).

${ }^{22}$ Señalemos de paso que el término verdad tiene el mismo sentido. Es decir, como la cualidad de lo verdadero, aquello que hace verdadero lo verdadero, la esencia de lo verdadero; cosa que es frecuentemente olvidada y que confunde lo verdadero con la verdad.
} 
vislumbrar qué es la objetividad, por tanto, es necesario indagar en los objetos qué los hace ser tales.

Sin embargo, si los objetos se presentan como cosas en sí, independientes del sujeto, suficientes y completas por sí mismas; y la conciencia se presenta como esencialmente diferente de los objetos, entonces surge la cuestión de cómo saber que cada conciencia individual tiene las mismas cosas. Es decir, si el ámbito de los objetos y el ámbito de la conciencia son dos esferas completamente diferentes, entonces cada individuo puede saber con certeza lo que está en su conciencia pero no puede saber con certeza lo que está en la conciencia de otra persona. Así, si añadimos que el lenguaje es derivado de lo que tenemos en la conciencia, entonces cada individuo tendrá su lenguaje privado y, por tanto, surge el cuestionamiento de cómo se realiza la comunicación. Estos son aspectos fundamentales del problema del solipsismo. La consecuencia epistemológica obvia del solipsismo es que el conocimiento, y no sólo el lenguaje, sería totalmente privado, sin posibilidad alguna de hacerlo común. Este pseudo-problema o tercer dogma del empirismo ha sido tratado por medio de la intersubjetividad de la siguiente manera. ${ }^{23}$

Primero, el concepto objeto es moderno y se asume que es eso al cual se dirige el pensamiento con la finalidad de conocerlo. Tanto el empirismo como el racionalismo aceptan que los objetos, las cosas

\footnotetext{
${ }^{23}$ Aquí llamamos al solipsismo pseudo-problema por el hecho de que desaparece desde el momento en que se asume que el punto de partida no es la separación entre objeto y sujeto, por un lado, y entre los sujetos, por el otro, sino que el supuesto fundamental y primero es la aceptación de la existencia de algo común tanto entre el objeto y el sujeto como entre los sujetos. Ahora, la aceptación de la existencia de algo común entre los sujetos es tácita y evidente desde el momento en el cual escribimos y hablamos, pues escribimos para alguien y hablamos para alguien, es decir, que el punto de partida es nuestra pertenencia a alguna comunidad, aspecto que ya ha sido puesto de relieve por el pragmatismo.
} 
por conocer, están en la conciencia. Sean, por una parte, sensaciones o percepciones impresas por algo externo a la conciencia; o sean, por otra parte, ideas innatas o fenómenos (resultado de la relación entre las intuiciones puras y las cosas en sí, en el sentido de Kant). Eso por conocer se encuentra en la conciencia, que también es un concepto moderno. Con esta postura se pretende resolver el problema del imposible acceso a las cosas en sí; entonces el conocimiento es de fenómenos, lo dado, evidencia fenoménica, o cualquier otro nombre que se prefiera darle.

Ahora bien, la exigencia según la cual el conocimiento consiste en proposiciones justificadas, requiere que aquello que está en la conciencia y que hace que los objetos sean objetos, sea lo mismo para toda conciencia. De lo contrario no hay garantía de que cada conciencia tenga lo mismo en ella y, por tanto, de que hable del mismo objeto. Para obtener esta garantía tenemos que asumir que las facultades de los sujetos son iguales, que son exactamente las mismas (sujeto trascendental kantiano) y son afectadas de la misma manera todas ellas por las cosas en sí. Así, las percepciones o sensaciones serían iguales en cada conciencia, puesto que lo externo las afecta de la misma manera; ${ }^{24}$ o nuestras ideas innatas o fenómenos serían los mismos, puesto que las formas subjetivas son las mismas.

Por consiguiente, en segundo lugar, según los presupuestos de las teorías de la verdad descritas anteriormente, la exigencia de que conocimiento sea proposición justificada y que el fundamento de lo que es objeto está en la conciencia, la objetividad consiste en la mismidad de cada conciencia, en la igualdad de cada sujeto (en cuanto ente cognoscente), en que cada sujeto posea las mismas facultades para cono-

\footnotetext{
${ }^{24}$ Este aspecto ya está expresado en el empirismo de Neurath (2000: 287-322), quien afirma que el lenguaje unificado es intersensorial e intersubjetivo.
} 
cer. A esta igualdad de los sujetos podemos llamarla intersubjetividad. Así pues, la objetividad consiste en esa intersubjetividad. ${ }^{25}$

En Kant esa intersubjetividad, que consiste de las intuiciones "tiempo" y "espacio" y la tabla de doce categorías agrupadas en cuatro grupos con tres categorías cada grupo, es una estructura estable y permanente. Pero las profundas críticas a que fue sometido el pensamiento kantiano por parte del empirismo de los siglos XIX y XX, llevaron a este empirismo a concebir esa intersubjetividad como una estructura cambiante y modificable, de modo tal que cada comunidad puede establecer su propia estructura intersubjetiva. La diferencia fundamental entre estas dos posturas es que para Kant la estructura no es producto de la convención sino a priori, mientras que para el empirismo posterior la estructura intersubjetiva sí es producto de la convención.

Con anterioridad hemos afirmado que las teorías de la verdad están guiadas por la actitud y la respuesta que se han tomado frente al escepticismo. ¿Cuál es la actitud del empirismo contemporáneo ante el escepticismo? La respuesta es de escándalo. El "escándalo" del empirismo es pretender dar respuesta a los argumentos del escéptico. Es decir, el empirismo aceptó los argumentos del escéptico como válidos. ¿Cuál es la respuesta del empirismo frente al escepticismo? Darse a la tarea de buscar un procedimiento que diera satisfacción al escéptico.

Lo que el empirismo aceptó fue lo siguiente: 1) que conocimiento es creencia justificada, lo cual es sinónimo de verdadera, ${ }^{26}$ 2) que la

\footnotetext{
${ }^{25}$ Véase por ejemplo: Hume, Kant, Dilthey, Neurath, Villoro y Apel, en sus textos ya citados.

26 Podría pensarse que Edmund Gettier ha demostrado que la justificación no implica de manera lógica la verdad de las creencias. Estoy de acuerdo que la justi-
} 
justificación es un procedimiento inferencial y 3) que la verdad es un carácter de las proposiciones. Sin estos presupuestos el escepticismo no tiene sentido. Es claro que el justificacionismo es la otra cara de la moneda del escepticismo. Dicho de manera directa, el justificacionismo no tiene sentido sin los presupuestos del escepticismo. Escepticismo y justificacionismo se presuponen recíprocamente.

\section{El significado de la pregunta ¿qué es la verdad? y la $\alpha \lambda \eta \theta \varepsilon \iota \alpha$ como determinante ontológico del ente que pregunta sobre la verdad}

\section{El significado de la pregunta ¿qué es la verdad?}

Veamos ahora una actitud y una respuesta diferentes frente al escepticismo. En lo que a continuación se expone seguimos el razonamiento que Martin Heidegger explica en su curso del semestre de invierno

ficación no implica la verdad, pero no es porque Gettier lo haya demostrado. Los ejemplos de Edmund Gettier omiten una condición de la cual Nicholas Rescher ha mostrado su necesidad: la información disponible para quien lleva a cabo la justificación. En los ejemplos de Gettier la información no está disponible para el individuo que realiza la justificación sino para un tercero. Por ejemplo, el ejemplo del profesor que intenta justificar la afirmación "por lo menos un alumno de mi clase tiene un auto Ferrari”, cuenta con la información siguiente: tal alumno de su clase maneja un auto Ferrari, tiene una factura (suponemos que la factura está a su nombre) y afirma tener un Ferrari. El ejemplo supone que el alumno en cuestión quería engañar al profesor y no posee un Ferrari. Esta información no está disponible para el profesor, es decir, el profesor no cuenta con evidencia alguna que contradiga la información que le permite creer que el alumno en cuestión tiene un Ferrari. Por el contrario, si el profesor tuviera alguna evidencia o indicio que le indicara que el alumno lo pretende engañar, entonces no podría estar justificado para sostener la afirmación "al menos un alumno de mi clase tiene un auto Ferrari”. Pero, ¿quién tiene la evidencia del engaño del alumno? Una persona distinta al profesor. En conclusión, los ejemplos de Edmund Gettier demuestran que nuestro 
de 1925-1926 impartido en Marburgo, que ha sido editado con el nombre Lógica, la pregunta por la verdad (2004).

El inicio del razonamiento es la pregunta ¿qué es la verdad? Desde la perspectiva escéptica parece que éste es un mal inicio, puesto que antes de preguntar ¿qué es la verdad? tendríamos que preguntar si existe la verdad. Recordemos que ésta es la pregunta correspondiente al primer punto de la formulación del escepticismo que expusimos anteriormente. Pero la propia formulación de la pregunta sobre la existencia de la verdad presupone como condición de posibilidad una comprensión de lo que es la verdad. Para poder fijar la pregunta de si algo existe o no, antes es necesario tener una comprensión de ese "algo", de lo contrario, no es posible captar el sentido de lo preguntado. ${ }^{27}$ Por lo tanto, se impone como primera la pregunta ¿qué es la verdad?

Por otra parte, si a la pregunta ¿existe la verdad? respondemos de manera afirmativa, no sólo presuponemos una comprensión de lo que sea la verdad, sino que estamos haciendo un enunciado "verdadero" sobre la verdad; de tal manera que, nuevamente, la respuesta a la pregunta supone ya una explicación previa de qué es la verdad. Y, finalmente, si a la pregunta ¿existe la verdad? respondemos de manera negativa ocurre lo mismo que con la respuesta afirmativa, hacemos enunciados verdaderos sobre el no ser de ser de la verdad. Entonces,

conocimiento es falible debido, en parte, a que la información disponible siempre es limitada, pero no demuestran que la justificación no implique la verdad. Esta condición de la información disponible siempre limitada ha sido analizada por $\mathrm{Ni}$ cholas Rescher. Por otra parte, Luis Villoro propone distinguir entre la definición de verdad y los criterios de verdad, porque son cosas distintas la verdad y la manera como nos percatamos de que nuestras creencias son verdaderas.

27 "Eso supone sin embargo que la explicación de lo que sea la verdad antecede a la de si la hay y a la de su posible captabilidad y comunicabilidad" (Heidegger, 2004: 26). 
la pregunta ¿existe la verdad? y su respuesta afirmativa o negativa supone antes la pregunta ¿qué es la verdad? ${ }^{28}$ Concluyamos de esto lo siguiente:

1. La pregunta ¿existe la verdad? siempre supone que antes se haya explicado la pregunta ¿qué es la verdad?, sea para su formulación y fijación, sea para su respuesta afirmativa o negativa.

2. Por tanto, como una cuestión de hecho (una cuestión fáctica) el punto de partida del problema o fenómeno de la verdad es la pregunta ¿qué es la verdad?

3. La actitud frente al escepticismo es no tomar en serio sus argumentos puesto que la pregunta de la cual parte no tiene sentido y, por tanto, la respuesta al escéptico ya no puede ser la búsqueda del procedimiento que justifique la existencia de la verdad. Ahora el punto de partida es la pregunta ¿qué es la verdad?, así se ha establecido el hecho que la verdad existe, pero es necesario indagar qué es.

Es necesario aclarar que la pregunta ¿qué es la verdad?, si es efectivamente una pregunta, una duda de la cual no se sabe la respuesta, no puede presuponer respuesta alguna, ni que la verdad sea correspondencia, coherencia o consenso (pues eso sería presuponer la

${ }^{28}$ Heidegger concluye el razonamiento de la siguiente manera: “a) la pregunta aparentemente primaria de si la verdad existe en general no es en absoluto la primera; b) es más, ella no es en absoluto objeto de una explicación con sentido, porque una explicación tal presupone ya siempre la posibilidad de la verdad.

"El preguntar cognoscente no puede en absoluto remontarse con su pregunta hasta antes de ese presupuesto fundamental de que existe en general la verdad. La reflexión se encuentra aquí en un límite.

"En este límite de la consideración y de la discusión, el afianzamiento de la consistencia de la verdad se resuelve en cierta manera por sí mismo. La amenaza para esta consistencia a cargo del escepticismo se hace inocua tan sólo en cuanto se da a pensar que el escéptico se contradice a sí mismo y de ese modo se anula" (2004: 26). 
respuesta a la pregunta) y, por tanto, tampoco puede presuponer el "lugar" de la verdad como siendo la proposición. Estos son asuntos que sólo se pueden contestar una vez que se ha dado una respuesta satisfactoria a la pregunta primera de ¿qué es la verdad? Es decir, la pregunta ¿qué es la verdad? no está orientada a justificar proposiciones, porque no se sabe si la verdad es justificación, precisamente es eso lo que se pregunta qué es la verdad. Veamos cómo es posible responder esta pregunta. ${ }^{29}$

\section{La $\alpha \lambda \eta \theta \varepsilon \iota \alpha$ como determinante ontológico del ente que pregunta sobre la verdad}

Vamos a analizar en qué consiste la verdad de las proposiciones con la finalidad de aclarar su presupuesto y elucidar las condiciones de posibilidad de esos presupuestos y, así, mostrar qué es lo que se requiere

${ }^{29}$ No es fácil determinar el momento preciso cuando Heidegger percibió el concepto de $\alpha \lambda \eta \theta \varepsilon \iota \alpha$ como fundamental para la filosofía, pero al menos podemos señalar algunos lugares en los cuales ya está claramente pensado la comprensión de la $\alpha \lambda \eta \theta \varepsilon \iota \alpha$ en un nivel ontológico. Heidegger, ya desde 1922, en el informe Natorp: Interpretaciones fenomenológicas de Aristóteles, señala que la comprensión autentica de la aletheia es fundamental para la filosofía. Posteriormente analiza el problema de la verdad y la $\alpha \lambda \eta \theta \varepsilon \iota \alpha$ en el curso del semestre de invierno de 1924-1925 que se ha publicado como Platon: Sophistes. Luego, trata el tema en el curso del semestre de invierno en Marburgo de 1925-1926 que fue publicado con el título: Logik. Die Frage nach der Warheit. Después, 1927, en Ser y Tiempo también sintetiza lo expuesto en extensión en los anteriores cursos. A partir de este momento Heidegger comprende y expone la $\alpha \lambda \eta \theta \varepsilon \iota \alpha$ como un determinante del Dasein. Pueden verse sobre todo Introducción a la filosofía curso de 1928-1929 (Einleitung in die Philosophie); La esencia del fundamento de 1929; La doctrina de Platón sobre la verdad, publicado en 1942, pero ya presentado como conferencias en 1930-1931 y 1933-1934 y leído para un grupo restringido en 1940; Parménides, curso de 1942-1943; La esencia de la verdad de 1943 y, finalmente, el curso de invierno de 1966-1967 titulado Heráclito. La lista no agota todos los lugares donde el autor trata el tema. 
para estar en posición de poder responder a la pregunta ¿qué es la verdad? El presupuesto de la verdad de las proposiciones es que necesariamente esa verdad tendrá que ser una correspondencia, ya sea que lo que debe corresponder sean las proposiciones y los objetos (verdad como verificación), o que la correspondencia debe establecerse entre las proposiciones con otras proposiciones (verdad como coherencia y como consenso).

Evidentemente, correspondencia es una relación y, por consiguiente, como toda relación, conecta dos términos: en nuestro caso proposiciones y objetos o proposiciones y proposiciones. ${ }^{30} \mathrm{El}$ primer caso es el que nos interesa, pues en él se lleva a cabo la elucidación de la relación entre el pensamiento y las cosas. ${ }^{31}$ Por tanto, las condiciones de posibilidad de la relación de correspondencia, para relacionar proposiciones y objetos, son que las proposiciones sean formuladas y que los objetos estén presentes.

Puesto que nuestra costumbre puede llegar a convertirse en una segunda naturaleza y estamos acostumbrados, desde hace más de dos

\footnotetext{
${ }^{30}$ La relación de correspondencia puede establecerse en diferentes respectos. Por ejemplo, la correspondencia entre proposiciones con proposiciones corresponderá en cierto respecto (el significado, la coherencia o algún otro); pero la correspondencia entre las proposiciones y los objetos corresponderá en un respecto muy diferente del anterior, pues entre estos términos — proposiciones y objetos - no existe nada físico que les sea común. Pero también podemos hablar de correspondencia entre cosa y cosa.

${ }^{31}$ Es necesario aclarar qué significa concordar, corresponder o coincidir: "Hablamos de coincidencia con distintos significados. Decimos, por ejemplo, ante la presencia de dos monedas de cinco marcos sobre la mesa: coinciden recíprocamente. Ambas se corresponden en la unidad de su aspecto. Por eso tienen éste en común, y por eso son iguales en ese respecto. Además, hablamos de coincidencia cuando decimos, por ejemplo, de una de las monedas presentes de cinco marcos: esta moneda es redonda. En este caso el enunciado coincide con la cosa. Ahora la relación no existe entre cosa y cosa, sino entre un enunciado y una cosa" (Heidegger, 1987: 14).
} 
mil años, a concebir la verdad únicamente como la correspondencia entre proposiciones y objetos, no resulta fácil dirigir el pensamiento hacia otra dirección respecto a la verdad. Por ello, es importante procurar exponer lo más claramente posible la perspectiva de la verdad guiada por el hecho de su existencia e iniciar su elucidación con el intento de responder a la pregunta ¿qué es la verdad? Con la pretensión de aclarar lo más posible esta perspectiva resumimos a continuación la secuencia de nuestro razonamiento.

1. Las teorías de la verdad ubican la verdad en las proposiciones.

2. Si el lugar de la verdad está en las proposiciones, entonces se presupone que la verdad es la correspondencia, particularmente, entre las proposiciones y los objetos.

3. La verdad como correspondencia, por tanto, tiene como condition sine qua non que previamente estén presentes las proposiciones y los objetos.

4. Por consiguiente, ¿la verdad como correspondencia depende de cómo se "presenten" los objetos?

Así, la ubicación de la verdad ha pasado de las proposiciones a los objetos. ${ }^{33}$ Es decir, la pregunta ahora es ¡cómo se presentan los entes? Sin embargo, la pregunta tiene ciertos determinantes, porque no se pregunta de manera genérica ¿cómo se presentan los entes?, sino que se pregunta tácitamente cómo se presenta a alguien. ¿Quién es ese alguien? No puede ser otro que aquel que se pregunta ¿qué es la verdad? Así, el primer contraste con los puntos de partida de las teo-

\footnotetext{
33 "El enunciado es como tal un enunciado sobre el objeto; el enunciado mismo encierra una relación con el objeto. Hemos llamado a esa relación la relación veritativa, simplemente para indicar que es en esa relación con el objeto donde aparece en primer plano la verdad" (Heidegger, 1996: 74).
} 
rías de la verdad es la diferencia de ubicación de la verdad. La verdad ubicada en las proposiciones forzosamente tiene que comprenderse como correspondencia. Pero si ahora se ubica la verdad en los objetos, en la presentación de los objetos, entonces no puede comprenderse como correspondencia, puesto que, como hemos visto, la correspondencia es una relación e involucra dos términos, situación que no se encuentra en la presentación de los objetos.

Por otra parte, aquel que pregunta ¿qué es la verdad? es él mismo un ente, por tanto, se pregunta también cómo se presenta este ente. Preguntar, ¿cómo se presentan los entes? es preguntar cómo existen, cómo son; de la misma manera que preguntar ¿qué es la verdad? es preguntar cómo existe, cómo es la verdad. Por tanto, se pregunta cómo es el ente que pregunta sobre la verdad. Preguntar ¿qué son los entes?, ¿`cómo son? es preguntar sobre su ser, por lo cual la pregunta ¿qué es la verdad? se ha remitido a cómo es el ser de aquel ente que pregunta ¿qué es la verdad? De esta manera, Heidegger ubica el fenómeno de la verdad en un nivel ontológico. ${ }^{34}$ No olvidemos que este nivel se muestra como condition sine qua non de la verdad como correspondencia.

Tampoco debemos olvidar que en esta segunda actitud y respuesta ante el escepticismo se ha mostrado que el punto de partida del problema de la verdad no es, como el escéptico pretende, la pregunta ¿existe la verdad? Sino que en su lugar se ha impuesto la pregunta ¿qué es la verdad?, esto es ¡cómo es la verdad? Esta pregunta tiene el mismo sentido que la pregunta a la cual nos ha remitido ésta: ¿qué son los entes?, ¿cómo son los entes? Particularmente, ¿̇cómo es el ente que pregunta sobre la verdad? Por tanto, estas preguntas no son guiadas

\footnotetext{
${ }^{34}$ Para esta argumentación del paso de las proposiciones a los objetos y de estos al ser del ente que pregunta, véase: La esencia de la verdad y Ser y Tiempo, introducción.
} 
por la perspectiva de "justificar" la existencia de la verdad o de los entes, sino por la de mostrar cómo son.

En este contexto, esto es, tomando como punto de partida la pregunta ¿qué es la verdad?, la cual nos ha remitido a la pregunta ¿cómo es el ente que formula esa pregunta? y que no está dirigida a justificar la verdad, en este contexto, repetimos, es que Heidegger ubica la concepción de $\alpha-\lambda \eta \theta \varepsilon \iota \alpha$. Esta concepción está dirigida a responder la pregunta ¿cómo se presentan los objetos, los entes? particularmente ¡cómo se presentan al ente que pregunta sobre la verdad?

Específicamente, la concepción de la $\alpha-\lambda \eta \theta \varepsilon \iota \alpha$ está dirigida a responder cómo es el ente que pregunta sobre la verdad y hemos establecido que esta pregunta se ubica en un nivel ontológico, de tal manera que la $\alpha \lambda \eta \theta \varepsilon \iota \alpha$ es una determinante ontológica del ente que pregunta ¿qué es la verdad? Es decir, la presencia de los objetos es condición de posibilidad de la correspondencia entre las proposiciones y esos objetos (la verdad como correspondencia o proposicional), y para que los objetos se presenten al ente que quiere establecer esa correspondencia, este ente tiene que tener algún tipo de acceso a la presencia de esos objetos. Este acceso es la $\alpha \lambda \eta \theta \varepsilon \iota \alpha{ }^{35}$

Dicho en otras palabras, el ente que pregunta sobre la verdad está constituido ontológicamente por una apertura ante la presencia de los objetos, esa apertura es la $\alpha \lambda \eta \theta \varepsilon \iota \alpha$. Esta es la determinación de su ser que le permite relacionarse con el mundo, mundo del

\footnotetext{
${ }^{35}$ En el parágrafo 44 de Ser y Tiempo Heidegger define la verdad como "un modo de ser del Dasein". Ese modo de ser lo llama en alemán Unverborgenheit. El término es intraducible al castellano, sería algo tan grotesco como "no-ocultariedad", calidad de no estar oculto. Frecuentemente se traduce como des-ocultamiento o desencubierto. "El sernos manifiesto el ente es un Unverborgenheit. Desocultamiento se dice realmente en griego $\alpha \lambda \eta \theta \varepsilon \iota \alpha$, que se suele traducir por verdad sin saber muy bien lo que se dice" (1996a: 87).
} 
cual él forma parte. Por tanto, puesto que la correspondencia entre proposiciones y objetos depende de la presencia de esos objetos, pero como estos sólo se presentan en un mundo, entonces, antes de que sea posible hacer corresponder proposiciones y objetos, el ente que pretende llevar a cabo esa correspondencia tiene que estar ya en un mundo. ${ }^{36}$

No vamos a desarrollar aquí el concepto de mundo pues ello requiere mayor espacio y no es el centro de lo que en este trabajo pretendemos exponer, baste con que quede indicado como asunto relacionado con el problema de la verdad. Sin embargo, para finalizar nuestra exposición sobre la verdad desde el punto de vista de la segunda actitud y respuesta al escepticismo, exponemos de qué forma pueden relacionar los distintos niveles en que la verdad puede ubicarse.

Comencemos con la enunciación de los niveles en los cuales se puede ubicar la verdad:

1. Verdad ontológica es la ubicación de la verdad en la interpretación o comprensión del ser de los entes.

2. Verdad óntica es la ubicación de la verdad en la propia presencia - patencia- de los entes.

3. Verdad proposicional donde se ubica la verdad como la correspondencia entre proposiciones y objetos.

La relación que es menester determinar entre estos tres niveles en que podemos ubicar la verdad, es la relación de enraizamiento que

36 Estar o ser en un mundo supone ya dentro de éste muchos comportamientos previos del ser humano ante las cosas que se le presenta en ese mundo, a constituirse en un sujeto cognoscente y, por tanto, en esos comportamientos previos el ser humano no concibe las cosas del mundo como objetos por conocer. Por ello, en este nivel no se presenta el problema de ¿qué es la objetividad? La objetividad es una comprensión derivada de las cosas. 
existe entre la primera y la segunda, y entre la segunda y la tercera $y$, por tanto, entre la primera y la tercera. Iniciamos con la segunda pues, evidentemente, constituye el nexo entre las otras dos.

La verdad óntica constituye lo que debe estar dado previamente y que es condition sine qua non de la relación de correspondencia de las proposiciones y los objetos y, por tanto, esta verdad, por ser previa a la verdad proposicional, es ante-proposicional; es decir, es no discursiva, pero es condición de posibilidad de lo discursivo. La verdad óntica es, pues, el acceso a los entes, es la patencia de los entes, el tener presentes los entes, la apertura a que los entes se muestren como tales. ${ }^{37}$

La verdad proposicional, la que consiste en la relación de correspondencia entre las proposiciones y los objetos, depende directamente de la verdad óntica; en efecto, requiere la previa presencia de los objetos. Esta verdad, por tanto, es discursiva, es predicativa. En este tipo de verdad es donde podemos colocar un procedimiento justificatorio de las proposiciones, el cual está soportado por la verdad óntica, quien quizá no admita ningún proceso de justificación. ${ }^{38}$

Finalmente, la verdad ontológica constituye el ámbito en el cual y por el cual es posible captar las diversas interpretaciones y/o com-

37 "La correspondencia del nexus [de las proposiciones] con el ente, y conforme a ella, su acuerdo, no hacen primariamente accesible el ente, como tal. Este, como el posible 'sobre el cual' de una determinación predicativa, debe ser evidente antes de esta predicación y para ella. La predicación para llegar a ser posible, ha de poder afincarse en un hacer manifiesto, que tiene carácter no predicativo. La verdad propocisional está enraizada en una verdad más originaria, en la patencia ante-predicativa del ente que llamaremos verdad óntica" (Heidegger, 1987: 67, las cursivas son del original).

38 "Tenemos, pues, que mostrar dos cosas: primero, que la verdad, que tradicionalmente se entiende como propiedad del juicio, como aedequatio intellectus et rei, se fundamenta en algo distinto, es decir, que lo que en el juicio se entiende como verdad es ciertamente una determinación genuina, pero que la interna posibilidad de la verdad radica en algo más original y es esto más original lo que, en segundo lugar, habremos de caracterizar" (Heidegger, 1996a: 61). 
prensiones del ser de los entes. Según Heidegger, los objetos, los entes, no se presentan de una única manera al ente que pregunta sobre la verdad. Las diferentes formas en que se presentan esos objetos, de los entes, depende de una comprensión tácita o expresa — generalmente tácita - del ser de esos entes. La elucidación de esa comprensión del ser de los entes, en cada caso, es la verdad ontológica. ${ }^{39}$

Entonces, la verdad ontológica es previa a la verdad óntica y ésta es previa a la verdad proposicional, por lo cual, por transición, la verdad ontológica es previa a la verdad proposicional. Pero quizá ni la verdad ontológica ni la verdad óntica admitan un proceso de justificación, proceso que, quizá, únicamente pueda ser posible en la verdad proposicional. Esto debe hacernos pensar en los límites de un proceso de justificación y en los límites de las teorías de la verdad fundadas en la perspectiva justificacionista.

\section{Conclusión}

La problemática de la verdad, según mis luces, oscila entre lo que hemos llamado la orientación de las teorías de la verdad y la orientación ontológica que concibe la verdad como $\alpha \lambda \eta \theta \varepsilon \iota \alpha$. Probablemente la primera orientación esté guiada por el principio de razón suficiente, según el cual nihil est sine ratione, y la segunda por el cuestionamiento de dicho principio. Ya Hume cuestionó el principio de razón suficiente en su Tratado, pero su empirismo, inevitablemente, lo condujo al escepticismo. Kant, por su parte, hizo del principio "una condición

\footnotetext{
39 "Pero lo óntico patente acontece en un encontrarse en medio del ente impulsivo y conforme al temple de ánimo, y en las actitudes hacia el ente co-fundamentadas en la aspiración y en la volición. Pero ni aún éstas son capaces de que se interpreten como antepredicativas o como predicativas. De hacer accesible al ente en sí mismo, si su patencia está ya de antemano iluminada y orientada por una comprensión del ser del ente" (Heidegger, 1981: 67-68).
} 
de posibilidad a priori de la experiencia". Las críticas lanzadas contra la distinción entre cosa en sí y fenómeno, contra la distinción entre juicios sintéticos a priori y juicios analíticos y, por tanto, contra la noción kantiana de "intuición", tuvieron como resultado en los críticos de esas nociones un retorno a Hume. Este retorno tuvo dos vertientes: 1) vía empirismo, que tiene como resultado el inevitable escepticismo; y 2) vía convencionalismo, que también tiene como resultado una variante del escepticismo, el relativismo.

Según la orientación de las teorías de la verdad tiene como resultado inevitable la idea del relativismo de la objetividad, puesto que la objetividad es intersubjetividad y ésta es relativa a las diferentes comunidades epistémicas, pues - por las críticas antes mencionadas a las nociones kantianas - la intersubjetividad es cambiante, es convencional tácita o expresamente. De tal manera que la verdad es relativa a la intersubjetividad de los diversos grupos de sujetos. Pero esta relatividad de la verdad es contraria al presupuesto de la orientación de las teorías de la verdad, según el cual nibil est sine ratione.

La orientación ontológica, por su parte, refuta el escepticismo mediante el análisis de la primacía de la pregunta ¿existe la verdad? mostrando que el escéptico se contradice a sí mismo y que la pregunta que se impone como original es ¿qué es la verdad? El sentido y el significado de esta pregunta no están dirigidos a justificar la existencia de la verdad, la pregunta misma supone la existencia de la verdad. El significado de la pregunta es ¿cómo es la verdad? y su sentido es dirigirse a ubicar el lugar y explicar "la manera de ser" de las condiciones de posibilidad de justificar $(\alpha \lambda \eta \theta \varepsilon\llcorner\alpha)$, es decir, el modo en que existe eso que ya la pregunta supone que existe; condiciones de posibilidad cuya existencia no es posible justificar.

La ubicación y la manera de ser de aquello cuya existencia supone la pregunta ¿qué es la verdad? es: su lugar está en la "naturaleza" del ente que formula la pregunta y su manera de ser es la $\alpha \lambda \eta \theta \varepsilon \iota \alpha$. Naturaleza y $\alpha \lambda \eta \theta \varepsilon \iota \alpha$, de las cuales no es posible formular la pregunta 
que se dirija a dar una justificación de su existencia, pues eso sería como preguntar ¿por qué debemos ser racionales? Por tanto, parece que la orientación ontológica impide formular las preguntas que se dirijan a justificar la existencia de eso que se pregunta.

Así pues, tanto la orientación de las teorías de la verdad como la orientación ontológica, implícita o explícitamente, cuestionan el principio de razón suficiente. Ello nos conduce a la necesidad de tener que preguntarnos ¿omne ens habet rationem? El problema de la verdad, por tanto, nos ha conducido no sólo al cuestionamiento del principio de razón suficiente, sino también al problema de la naturaleza del ente que pregunta sobre la verdad.

\section{Bibliografía}

Apel, Karl-Otto, 1991, Teoría de la verdad y ética del discurso, Paidós, Barcelona.

Austin, John Langshaw, 1964, “Truth”, en G. Pitcher (comp.), Truth, Prentice Hall, Engle Wood Cliffs.

Guignon, Charles. B., 1983, Heidegger and the problem of knowledge, Hackett, Indiana.

Coffa, Alberto, 1991, The semantic tradition: from Kant to Carnap, Cambrige University Press, Cambridge.

Descartes, René, 1960, Discours de la méthode, Mignon, París.

, 1971, Reglas para la dirección del espiritu, Porrúa, México.

Dilthey, Wilhem, 1978, Hombre y mundo en los siglos XVI y XVII, FCE, México.

Gettier, Edmond L., 1963, "Is justified belief knowledge?", en Analysis, vol. 23, pp. 121-123.

Goldman, Alvin, 1967, "A casual theory of Knowing”, en The Journal of Philosophy, LXIV, 12, 22 de junio, pp. 357-372.

Heidegger, Martin, 1960, "La época de la imagen del mundo", en Sendas perdidas, Losada, Buenos Aires.

, 1973, Kant y el problema de la metafisica, FCE, México. 
, 1980, Ser y tiempo, FCE, México.

, 1987, "De la esencia del fundamento", en ¿Qué es metafísica? y otros ensayos, Siglo Veinte, Buenos Aires.

, 1987a, "La esencia de la verdad", en ¿Qué es metafísica? y otros ensayos, op. cit.

, 1995, Qu'est-ce qu'une chose?, Tel Gallimard, Francia.

, 1996, "La question de la technique", en Essais et conférences, Tel Gallimard, París.

, 1996a, Introducción a la Filosofía, Frónasis, Madrid.

, 1996b, "Science et meditation", en Essais et conferences, op. cit.

2004, Lógica. La pregunta por la verdad. Alianza, Madrid.

Helmholtz, Herman Von., 1977, "On the origin and significance of the axioms of geometry”, en Schlick, M. (ed.), Epistemological Writings, Reidel Publishing Company, Dordrecht.

Hume, David, 1998, Tratado de la naturaleza humana, Tecnos, Madrid.

Klein, Jacob, 1968, Greek Mathematical Thought and the Origin of Algebra, Dover, Nueva York.

Mach, Ernst, 1908, La connaissance et l'erreur, Ernest Flamarion, París. , 1947, Análisis de las sensaciones, Daniel Jorro, Madrid. , 1960, The Science of Mecanics, La Salle / The Open Court, Chicago.

Musil, Robert, 1980, Pour une évaluation des doctrines de Mach, Presses Universitaires de France, París.

Neurath, Otto, 2000, "Sociología en fisicalismo", en Ayer, A. J., El positivismo lógico, FCE, México, pp. 287-322.

Poincaré, Henri, 1970, La science et l'hypothése, Ernest Flammarion, París.

Quine, Willard van Orman, 1962, Desde un punto de vista lógico, Ariel, Barcelona.

Rescher, N., 1991, La racionalidad, una indagación filosófica sobre la naturaleza y la justificación de la razón, Tecnos, Madrid.

Schlick, M., 1981, "Positivismo y realismo”, en Ayer, A. J., El positivismo lógico, op. cit., pp. 88-114. 
, 1987, General theory of knowledge, Springer, Nueva York.

Tarski, Alfred, 1944, "The semantic conception of truth", en Philosophy and Phenomenological Research, vol. IV, reeditado en Freilg y Sellars (comps.), Reading in philosophical analysis, Appleton Century Craft, Nueva York, 1949, pp. 52-84.

Turró, Salvio, 1985, Descartes: del hermetismo a la nueva ciencia, Ántrophos, Barcelona.

Villoro, Luis, 1980, Saber, creer y conocer, Siglo XXI, México. 